\title{
Small Satellites Constellations and Their Impact on CBRNe Management in Africa
}

\author{
Donato Morea $^{1 *}$, Giacomo Primo Sciortino ${ }^{2,3}$ \\ ${ }^{1}$ Department of Mechanical, Chemical and Materials Engineering, University of Cagliari, Via Marengo, 2, 09123 Cagliari, \\ Italy \\ ${ }^{2}$ Pegaso International, Smart City Malta, Unit 605 SCM01, Ricasoli, Kalkara SCM 1001, Malta \\ ${ }^{3}$ Agenzia Spaziale Italiana (ASI), Via del Politecnico, snc, 00133 Rome, Italy
}

Corresponding Author Email: donato.morea@unica.it

https://doi.org/10.18280/ijsse.110412

Received: 10 January 2021

Accepted: 16 July 2021

\section{Keywords:}

digital divide, microsatellites, project financing, satellite TLC, small satellites

\begin{abstract}
A wave of small satellites massive constellations, in the range of hundreds of units each, is progressively populating the Low Earth Orbit (LEO) with a low-price, and varied, offer of Telecom (speed band) and Earth Imaging services (Starlink, Planet, One Web, etc.). It is a market - driven trend based on new satellite interlocking technologies, which cut down the supplier costs of launch and in orbit operations compared to the traditional technology based on big (and much heavier) geostationary satellites operating at high altitudes. This is a disruptive phenomenon especially for the developing world, where such vital services have always been hard to access, and their use therefore remained scarce, not consolidated, or even completely missing. Among these, Emergency management is definitely crucial. The geographical focus of this study is Africa and it deals not only with Institutional PRS users but with a wider potential context (corporations, private subjects, etc.). It clearly appears that a general degree of "Country readiness" toward Space technology and organization is necessary for these initiatives to take place. This can be achieved through certified international cooperation.

The authors then, based on an estimated demand Model for services with their relative pricing corresponding to a cost-designed constellation of small microsatellites, presented already, among other, at several International Astronautical Federation(IAF)Symposia on Space Economy, simulate the resulting type of services available: TLC by band types and relative upload and download rates, Earth imaging by refresh rates and optical quality and resolution, Ground segment configuration for signal backhauling and user terminal receiving.. This info isapplied to a specific African Country case (Nigeria) whose significance emerged over other Countries after the application of comparative grids. Finally, an insight on the specifically configuration of services for Chemical, Biological, Radiological, Nuclear, and Explosive (CBRNe) like management by local users, both maritime and land, with the relative costs, is offered. This is consequently left open for follow ups and discussion, due to the customer - design, project financing approaches of this Model programme.
\end{abstract}

\section{SPACE SERVICES TO EARTH FOR EMERGENCY MANAGEMENT- THE FOCUS ON AFRICA}

According to the 2030 "Space Agenda" space technologies and applications are a crucial enabler of contexts security/safety and therefore "growth" in the Developing Countries but it is also fundamental that the access to these technologies be gained through virtuous and progressive international cooperation cycles. Otherwise, the extreme technical and organizational challenges implied, along with a pressing need of those Countries - especially for Telecommunication (TLC), Localization and navigation (NAV), Earth Observation (EO) services, to face digital divide, and gaps in education, healthcare, security, land and sea management - risk to create bottlenecks and polarize, rather than expand and integrate, the formation of these new competencies. This pitfall is clearer now that the new disruptive technologies of small satellite systems allow a considerable reduction of user prices, compared to the traditional offer based on heavy geostationary satellites.

Having Africa in the focus, the authors recently estimated that in the 2020s there could be a sizeable increase in the use of satellite - based services in the Continent, and particularly in TLC. In fact, the demand for web connections is strongly and steadily growing, quicker than in the rest of the World. This trend is mostly due to mobile networks, which in turn are less depending on traditional heavy and costly infrastructure than on cabled networks. Moreover, the "new generation" of low-cost/low-orbit small satellites, really appears more competitive than the traditional geostationary constellations, and obviously the cabled grids; this competitiveness is highlighted by the hard natural and social conditions of the Continent, which penalize by definition diffused land infrastructure. However, TLC is not the only growing demand area. In addition, the demand for various satellite EO services is on the increase, for land monitoring, security, urban and rural management, etc. [1].

Consider then that the supply of small satellite EO services 
has already really started in the Continent and reached market proportions, unlike TLC services. This is mainly driven by companies like the US' Planet Labs, which offer cheap medium resolution $(2-10 \mathrm{~m})$ images to a varied clientele, worldwide, and is progressively eroding the shares of other big suppliers such as Digital Globe, Airbus, etc. Some global governmental suppliers - EU Copernicus, US Landsat - also have a role in this trend, because they offer a wide portion of "free of charge" general images, thus stimulating the formation of initial demand. Total Africa's demand of EO data is foreseen to increase to almost 70M€ yearly in 2024 and the main role will be played by small satellites [2]. Regarding Planet Lab it could be usefully pointed out that to compensate a lower image resolution quality (their optical sensors are rather small) their system competitivity is very high regarding integration and ground assistance. Their worldwide Ground network is made up of 15 stations that guarantee both flight monitoring and net connection, with a fleet of 300 satellites, capable of an optimal refresh rate (5 times a day over the same area) in any place in the world [3]. Besides, this constellation covers 300MsqKilometers (almost the whole Earth's surface except beyond $80^{\circ}$ latitudes), with an image footage of ca. 20,000 sqKilometers, and a total processing download capacity of 7 Terabps/day for their customers [4].

\subsection{The peculiar challenge of context preparation in Satcom services}

On the other end, small satellites, in telecommunications, are still shy of going into full international operations. Relatively numerous launches have taken place already as accounted by the open UCS database on www.ucsusa.org/resources/satellite-database, but so far only to cover domestic offer of $5 \mathrm{G}$ services [5]. The US Bryce Space and Technology 2012-17 Study on small satellites launches, accordingly, reports in 2017 that commercial EO ones are largely prevailing over TLC and other areas, to the height of $85 \%$ of the yearly total of 335 launches.

Over the entire period about 1,000 launches of small satellites occurred, which was a lower than expected performance [6]. The reason of this delay is that communication satellites, the real potential market driver, require more extensive and careful preparation of their operations, than EO satellites; this is due to the higher rigidity of their service structure, requiring a more extended and careful preparation of the massive users' end. EO outputs instead get widely elaborated, distributed, trough complex and already existing ground networks, including the supportive effect of free of charge image ranges produced and publicly supplied by Governmental systems (e.g. Copernicus in the EU, Landsat in the USA).

What we observe in fact is, from the current evolution of satcom initiatives such as the US StarLink or the troubled OneWeb (now sold from USA to the UK), not to mention Hongyan and Huawei in China and Korea, that they finally came at the threshold of international market access after long precursory campaigns, and dealing with mainly strategical and institutional obstacles. Because ultimate access to operations depends on the target Countries' general "readiness" to host and use those hi-tech communication systems.

Some secondary pitfalls can derive, to say the truth, from technological and financial aspects. For one, the increasing risk of space debris creation by possible collisions between those new space vehicles, which because of cost efficiency are sent to fly in low-Earth orbit (LEO), and in formations of hundreds of units. To be more precise on this point, the 3 "top scorers in terms of constellation populations are currently Starlink (more than 1,000 units), Planet (300), OneWeb (150). They all plan to increase strongly these numbers in the next years. Imagine, for comparison, that the biggest constellation of ordinary satellites previously in orbit was the US Iridium, composed of "only" 66 units [7]. Anyway, the latest models of small satellites carry risk mitigating tools, in that following the UN COPUOS (Committee on the Peaceful Use of Outer Space), such as positioning sensors, and are made of special materials. This means, respectively, that they can be tracked and guided until their end of life and final disposal, and have almost $100 \%$ probability to be completely destroyed at reentry into the atmosphere. These solutions might become more useful soon if insurance companies decide to enter the business of debris risk coverage [8].

Again, various technical problems arise by definition in any systems which apply brand-new technology. In our case, one sensitive component could be the crucial interlocking radio communication system onboard each fleet unit. This system is in fact what makes it possible for "moving" units to supply a steady service for terrestrial users. But, for instance, what is the real endurance of COTS (large scale commercial) components of the onboard payloads over the entire expected operational life of the vehicle? Given the diverse possible malfunctioning originating is such complex endeavours, these constellations are conceived with robust self-repair and readjustment-compensation procedures. See, for instance, the protocols of immediate replacement by "in orbit injection" of a new satellite, in case of serious failures of an operational one, or the redundancy of inter communication devices between satellites (radio and optical together).

From the economic point of view, the main issues are not strictly financial, because so far these ventures are fully based on equity capital from big global corporations, such as Google, Coca Cola, PayPal, Amazon, etc. in search of high return rates. They are, rather, connected to the growing reactions and countermeasures put in place by the pre-existing traditional competitors, which progressively erode the initial targeted profit margins. Such as new, lighter and more cost - efficient satellites (e.g. with the possibility to lengthen their operational life by in orbit refueling).

\section{AN EVALUATION OF "COUNTRY READINESS» FOR PUBLIC AND PRIVATE INVESTMENTS IN AFRICA FOR THE USE OF SATELLITE SERVICES}

\subsection{Evaluating space readiness in the Continent - the index of oriented Official Development Assistance - ODA}

Thus, something is still holding back, and mostly in the TLC area, the innovative worldwide transition of satellite services to the low weight, low orbit, low cost paradigm, and Africa is especially involved. We therefore felt it necessary to conduct a deeper analysis of the possible reasons, also considering that, for a series of already explained facts, this Continent has all the characteristics to make these technologies an absolute and ideal solution. Our investigation moves of course inside the "core" of the problem, which, as stated previously, is the readiness of these Countries, to host and exploit any type of space technology, from the strategical and institutional point of view. Just consider the educational level necessary to 
prepare and maintain a local qualified workforce, or the complexity of international procedures and security permits related to the use of high radio frequencies or aerial imaging supplied by satellites.

Our investigation was based therefore on the application of readiness indexes to rank these Countries. Our first "index" was sought in the records of the IFIs (International Financial Institutions), because they represent the main, or even only, public (multilateral) source from which most of these Countries can get the necessary grant based funding to finance an appropriate "institutional strengthening" in such a technical field, as it happens in other domains. We refer particularly to the records of the main Entity operating in the Continent, which is the FMI - UN related World Bank (WB) Group, including the World Bank itself and the African Development Bank (AfDB). Specifically, the Country Space readiness evaluation unit, while analysing these records, has been the amount and trends of received (approved) Official Development Assistance (ODA) to support "space related" programmes. ODA, as by OECD DAC definition, is any type of bilateral or multilateral subsidized financial facility, whether a grant or a very low interest credit (bearing at least a $25 \%$ actualized grant element), or an equivalent guarantee (e.g. those issued by WB MIGA for project financing vehicle companies) approved for the benefit of a list of authorized Recipient Countries, which is updated every year upon their actual socio-economic conditions. At present (2019) all African Countries meet the eligibility requirements, because they are classified in one of the three categories, starting from the lowest development degree (LDCs), to the middle and upper ranking.

Such an indicator appears then very meaningful because these Countries, regardless of their individual rating about their development level (lower - LDCs, middle, and upper) represent in the above measurement a coherent statistical universe. Moreover, the role of the cited main International Financial Institutions - IFIs, as anticipated above, is exactly to support the promotion of basic policies regarding socioeconomic projects and programmes; and all this according to the Countries' vocations and primary needs, with the aim of ensuring their self-sufficiency and future stable development. Such Countries, in general, would find it difficult to cover these areas of intervention, because of institutional and financial weaknesses. Therefore, the fact that structural "space" investments for the relative systems deployments, including education, are part of the IFIs operation records, and the quality of these interventions, is a sort of certification that the "space services" approach gets an institutional backing also in perspective; and this is acknowledgement can be endorsed to the most authoritative levels of the whole international Community.

We must point out that such an indexed ranking was made by our research group and based on the projects description and total amounts of financing resulting by continental blocks in the general WB and AfDB detailed accountancy. Therefore, if a certain space content was always present, its share could not be easily established, apart from some descriptions (a minority or cases) where space services were clearly pivotal. In fact, the use of space refers mainly to TLC, EO or NAV satellite services applied to terrestrial programmes in transportation, rural and agricultural management, environmental control and disaster management [9].

Moving from this, we firstly extracted and observed these data over the period 2000-2015 noticing that space related
ODA going to Africa was $567 \mathrm{M} \$$ and the second continental recipient was Far East Asia, was $189 \mathrm{M} \$$. It was still a too limited amount to perform further study on a Space phenomenon, if we consider that the total ODA figure over the same period was $240 \mathrm{Bn} \$$.

But the second analysis on data covering the 2015 - 2018 period, revealed significantly increased numbers and figures for these space related projects, making them more than an "initiation". and justifying a more detailed study. In general the index still reveals too weak a propension to deal with space. The approved amount of such projects, concentrated in TLC and EO services, therefore does not seem to match its big potential role; the total figure of $\mathrm{WB}$ and $\mathrm{AfDB}$ together, considering the final year (2018) as a reference, is in fact worth for Africa 335,6 Million (M) \$. Although this is a record value, resulting from a continuous yearly increase, it still accounts for only $0.6 \%$ of this global "all-sectors" ODA, that is ca. 60 $\mathrm{Bn} \$$,

We could anyway observe some interesting trends regarding the directions of this funding: 1) LDCs Countries (lower per capita GNP classes of the Recipient Countries List) are largely prevailing. Among those there are massive flows toward very deteriorated socio-political situations (Democratic Republic of Congo, Mozambique and Rwanda), which reveals an emergency approach (drought, youth, disaster recovery) rather than a rational development policy. The only non-LDC Countries resulting in the Figures 1 and 2 are Kenya and the Ivory Coast, which concentrate sizeable subsidies respectively for the agricultural and forestry sector (EO remote sensing for environmental early warning and land management, TLC for rural e-government and production), and for the same again but with an emphasis on industrial processing activities; 2) the type of "space" content in these initiatives indicates a dominance of EO ( $53 \%$ of the funding), against TLC (47\%), even if in TLC Satcom initiatives we find the only cases ( 4 cases) where the space share is really high, substantial and pivotal, located in small, and presumably better strategically controllable, Countries (WB only projects), such as Comoros, Guinea Bissau, Malawi and Uganda. These 4 projects all fall in the "Transport and ICT" category and involve connectivity and e-services to support industrial development in the ECOWAS framework.

We could also deduct that the existence of these authoritative programmes was everywhere a mitigating factor against distortions and undue influences of mixed politicalcorporate interests toward specific national "space" choices, which too often lead, otherwise, to rigidities in newly created "markets", and difficulties to achieve a free, fair, competitive environment for venturers, and ultimately to ensure long lasting socio-economic benefits.

\subsection{Evaluating space readiness in the Continent - A survey on Country space "intensity" indexes}

Our second readiness index was taken from our previous studies is inspired to TU's IDI index (ICT Development Index), which combines three conceptual levels: access, use and skills.

It is a model declined into a multiple indicator specific for space, which in turn is an elaboration of the Porter's diamond framework, enriched with specific areas of evaluation. It encompasses the following elements: a) technological access, b) local demand, c) international integration and d) human and entrepreneurial factors' maturity toward sectoral growth [10]. 


\begin{tabular}{|c|c|c|c|c|c|}
\hline & \multirow[t]{2}{*}{ Country } & \multicolumn{2}{|c|}{ Approved amount by Type of space } & \multirow[t]{2}{*}{ Project description } & \multirow[t]{2}{*}{ Category } \\
\hline & & Satcom & Remote sensing - EO & & \\
\hline \multirow[t]{2}{*}{2015} & Kenya & & 2,5 & Disaster resilience and e-Government & Agriculture \& Forestry \\
\hline & Uganda * & 85 & & Regional development by e-services & Transport \& ICT \\
\hline Subtotal & & 85 & 2,5 & & \\
\hline \multicolumn{6}{|l|}{2016} \\
\hline & Central African Republic & 20 & & Economic recovery encompassing digital divide & Public Administration \\
\hline & Congo (Dem.Rep.) & & 8,03 & Disaster recovery hydrometeorology and climate & Urban and Rural Resilience \\
\hline & Rwanda & & 100 & Urban dev. Infrastructure (use of GIS) & Urban Transport \\
\hline Subtotal & & 20 & 108,03 & & \\
\hline \multicolumn{6}{|c|}{2017} \\
\hline & Guinea Bissau & 35 & & Gerographical speedband and cost reduction ECOWAS & Transport \& ICT \\
\hline & Kenya & & 280 & Kenya Climate and Smart Agriculture Infra & Agriculture \& Forestry \\
\hline & Malawi & 72,4 & & ECOWAS support connectivity and platforms & Transport \& ICT \\
\hline & Sierra L. & & 20 & Extractive industry technical assistance & Extractive \& Energy \\
\hline & Zambia & & 32,8 & Climate change, landscape management and rural & Agriculture \& Forestry \\
\hline Subtotal & & 107,4 & 332,8 & & \\
\hline \multicolumn{6}{|l|}{2018} \\
\hline & Burkina Faso & & 33 & Strengthening climate resilience and hydro warning & Urban and Rural Resilience \\
\hline & Central African Republic & 25 & & Reestablishment of basic fiscal management & Governance \\
\hline & Chad & & 44,6 & Climate resilient agriculture productivity enhancement & Agriculture \& Forestry \\
\hline & Comoros & 10 & & Multipurpose satcom for regional communication & Transport \& ICT \\
\hline & Ivory Coast & 70 & & Affordable speedband access and Infra TLC & Transport \& ICT \\
\hline & Mozambique & & 100 & Administration strengthening in land tenure and services & Urban and Rural Resilience \\
\hline Subtotal & & 105 & 177,6 & & \\
\hline Grand totals & 938,33 & 317,4 & 620,93 & & \\
\hline Notes: & * in bold characters proje & ith a high content 0 & of space components & & \\
\hline
\end{tabular}

Figure 1. World Bank approved ODA by Country in M€ (Africa) - Projects with space content

\begin{tabular}{|c|c|c|c|c|c|}
\hline & \multirow[t]{2}{*}{ Country } & \multicolumn{2}{|c|}{ Approved amount by Type of space } & \multirow[t]{2}{*}{ Project description } & \multirow[t]{2}{*}{ Category } \\
\hline & & Satcom & Remote sensing - EO & & \\
\hline \multirow{2}{*}{2016} & Congo (Dem.Rep.) & 436,1 & & Development of real digital economy in Central Africa & ICT Environment \\
\hline & Multinational & & 6,6 & Satellite weather info and early warning & Disaster Reduction \\
\hline Subtotal & & 436,1 & 6,6 & & \\
\hline \multicolumn{6}{|l|}{2017} \\
\hline & Multinational & & 56,5 & Feed Africa Continental support & Agriculture \& Forestry \\
\hline & Ivory Coast & & 126 & Belier Regional agroindustrial project & Agriculture \& Forestry \\
\hline Subtotal & & 0 & 182,5 & & \\
\hline \multicolumn{6}{|c|}{2018} \\
\hline & Chad & & 20,24 & Gerographical speedband and cost reduction ECOWAS & Transport \& ICT \\
\hline & Mozambique & & 15,51 & Kenya Climate and Smart Agriculture Infra & Agriculture \& Forestry \\
\hline & Mozambique & & 17,29 & ECOWAS support connectivity and platforms & Transport \& ICT \\
\hline Subtotal & & 0 & 53,04 & & \\
\hline Grand totals & 678,24 & 436,1 & 242,14 & & \\
\hline
\end{tabular}

Figure 2. African Development Bank approved ODA by Country - Projects with space content

This indicator has given positive results for a number of African States, which coincide with all those who can be considered "spacefaring", because they own at least one vehicle in Space: Algeria, Egypt, Ghana, Nigeria and South Africa. These Countries all qualified for having an average industrial access - see a) above -and diversified levels of the other strength factors: Algeria emerged for its human labour component, Egypt for its entrepreneurship and productive resources, Ghana in the quality of international political and entrepreneurial integration, Nigeria in the dynamism of its competitive domestic market. South Africa had emerged as a top scorer outsider, based on its superior level of technological advancement.

Unfortunately though, none of the above Countries had appeared in the first ODA index, not receiving sufficiently strong indications from the ODA orientation. Having still to select one Country for a specific temporary simulation to conclude this work, the second index seemed more suitable for our purposes, and the choice fell on Nigeria, despite the many issues of stability affecting this Country. Nigeria still emerged as the one with less relative weaknesses, so to say, toward the strong political uncertainty influencing Algeria; the recent technological "spatial" problems of Egypt regarding 2 main EO satellites (Egypt1 and Egypt2); the too little experience of Ghana with its first satellite launched only in June 2017; the peculiar position of S. Africa in the continental cultural panorama. Nigeria, besides, has successfully run, through NASRDA (National Space Research and Development 
Agency) the 2 Nigeria Sat-X and Nigeria Sat-2 UK built (Surrey Satellite Technology) multispectral / panchromatic EO microsatellites, and their relative environmental and security monitoring tasks.

\section{AN IDEAL CONTEXT SIMULATOR FOR PRIVATE INTERNATIONAL VENTURES IN TLC AND EO SATELLITE SERVICES FOR AFRICA - NIGERIA}

\subsection{A model programme for low cost sea/land satellite services - The Sea-Like Horizon Space Tutor (SHST)}

So, wanting to enrich our study about the ideal, and determining support that "space" cooperation can offer to the growth of developing Countries, and Africa in particular, with an efficient simulation, we decided to adapt a venture model for a low cost sea/land satellite services initiative which has already been used in similar scientific studies, the SHST - Sea Like Horizon Space Tutor [11] - and apply in detail all of its possible involvements of Nigerian subjects, whereas research theory should translate into market success. The SHST model is in fact designed to "stress" at most the attitude toward the reception of the initiative, and therefore quickly point out the possible obstacles to its success. To this aim, it is based on a "pure" private "project financing" scheme (no public interference of any kind involved), promoted by several investors, among which financing banks and equity shareholders, and centered in a "purpose company" and relative capital, to act as the legal and accounting center of the project.

The solid principles of "project financing" vouch for the meaningfulness of this "stress test". In fact, a project financing structure basically requires that a venture market risk be translated from the traditional financial guarantees (collateral, bonds, mortgages, etc.) to a multiple allocation mechanism among the various investors. The ultimate guarantee resides here in long term service purchase obligations by the final service users (and the right of immediate access to the generated turnover cash flow by the financing banks). The banks are in turn assimilated to the other stakeholders, with or without equity shares [12]. According to this approach, the presence of a client, or better of clusters of clients, interested in buying certain services at fixed prices, and willing to commit in the long term to this purchase, is the mainstay assumption. Just like in a "design to cost" initiative, the "product", which in this case is a small satellites' system, gets financed and built to meet exactly those requirements within a planned construction and launch time, as well as time for the start and duration of operations, and for debt repayment and profit generation. It will take in most cases, before such a deal is closed, a reiteration of reviews at both levels: on the side of the final users' requirements as well as on the technological and operational side, in reciprocal adaptations, to reach a balance between the market performance expectations and the relative technical and operational configuration of the service. This has been the case, several times already, for our simulator, which therefore has improved its economic and technical significance.

Regarding economic considerations, as they have already been introduced in this study, the business potential of small satellite multipurpose (TLC and EO together) constellations is very high. This is due to their great competitive edge, in turn based on seizable cost savings derived from innovative technological factors: low orbit functioning, light materials and vehicles, limited launch costs. Starting from this, and from the priority required by project financing methodology, the first phase of the SHST model construction was to define what prices the potential new clusters of clients were willing to pay, to replace the actual providers, if any. Both maritime and land users were involved in this first enquiry which brought to the design of a "bundle" of services, suitably consolidated in a single console with a highly standardized hardware and firmware for the user terminal, to be offered at ca. $50 \%$ of the prices actually incurred in the same service areas. This offer includes the company's proprietary equipment lending fee (e.g vessel terminals and antennas for the maritime users and user receivers for land users, except for community backhaul towers or other types of signal propagators if more than ordinary were necessary), whose level is anyway, thanks to standardization, relatively low.

Once a basic acceptance of those services and prices was obtained by the potential clusters of clients, complying with the requirements of a "project financing" initiative, and therefore their certified long-term fixed purchase commitment, the "design to cost" started. The actual configuration of this small satellites' system is as follows. The system's acronym represents the main features of this constellation of 20 microsatellites (ca. $30 \mathrm{~kg}$ each), that is their capacity to provide TLC speed band services to maritime users at all latitudes over the Atlantic and in "sea-like horizon" conditions - that is in the absence of conspicuous in-between rises of the terrain, whether natural or artificial(hills, mountains, high rise buildings)that the low signal angle could not overcome -as well as to all land users, way inland, up to $2500 \mathrm{~km}$ from the coasts. In addition, for maritime users, or authorized land users (see the national laws to preserve security and privacy) there is a near real time "ceiling" service of medium resolution daylight images of their covered area, supplied onboard each satellite by a set of fixed cameras, with a video streaming refresh of 10 seconds. In Table 1, you find more details of the SHST system. Its scheduled lifetime from date of launch is currently set at 5 years.

To sum it up, regarding potential maritime Nigerian users, which of course are only a limited portion of the total user Countries involved in the enquiries and scouting worldwide, this system can offer, to any type of clustered vessel (cargo, tankers, cruisers, fishing fleets, ferries, work ships and leisure boats) an integrated console of upload / download 10Mbps CIR rate TLC services (AIS, Point to Point for Headquarter interface, Internet media and Internet big data interactive, Telehealth and intervention of remote Center in $\mathrm{Ku}$ band), which represents a highly competitive alternative to the actual ordinary separate offer of similar services. For example, a big cargo ship or a tanker could get the bundle at a yearly price of $18.500 €$ and satisfy all needs, from ship's operations, to security vessel localisations - AIS, personnel connection needs and hw and firmware functioning insurance. What is important also, and available for maritime users only, is an additional near real-time daylight imaging package, supplied by the hovering satellites, with a sizeable ceiling of medium resolution $(2-10 \mathrm{~m})$ streaming (1 frame / $10 \mathrm{sec})$ shots, useful for example to assess the water surface conditions (pollution, contamination), the presence of fish shoals, the conditions of operational areas. Consider in comparison that the average price for similar images bundles is quoted at $1000 €$ and they are not obtainable in near real time, not in streaming mode (but as just a refresh of static images only a few times a day), on 
single shot basis and not repeatable at will within a ceiling, and finally with a footage of 100 sq kilometers only. Moreover, for higher resolutions price increases by correlation coefficient close to 1 [13-16].

Table 1. SHST microsat initiative - Main technical features

\begin{tabular}{|c|c|c|}
\hline System component & Characteristics & Notes \\
\hline Orbital configuration & $\begin{array}{l}\text { Ordinary geological rotation, hovering refresh } 2 \mathrm{~h} \text { at Equator. } 5 \text { Orbital } \\
\text { planes to cover evenly all latitudes [13]. }\end{array}$ & $\begin{array}{c}8 \text { satellites serve the equator, } 4 \\
\text { at each tropical longitude and } 2 \\
\text { at each subpolar. }\end{array}$ \\
\hline Satellites & $\begin{array}{l}20 \text { made in Italy vehicles with polymeric case weighing ca. 30kg each } \\
\text { (microsats), to place at } 1000 \mathrm{~km} \text { LEO (Low Earth Orbit) ca. and optimize } \\
\text { rule of } 2 \text { contemporary interlocked service for every user everywhere. } \\
\text { Data relay devices. Equipped with deorbiting - reorbiting supplementary } \\
\text { boosters to maneuver in case of need into different orbits. } 4 \text { units stock of } \\
\text { replacement is in the investment, } 2 \text { of which are already injected into } \\
\text { orbit to guarantee immediate replacement for serious failure. } \\
\text { Launch method under study: terrestrial mini launcher, Virgin } \\
\text { stratospheric plane, ISS, etc. }\end{array}$ & $\begin{array}{l}\text { Will ensure continuous self- } \\
\text { localization until demisal } \\
\text { destruction at atmospheric } \\
\text { reentry in known point. A } \\
\text { reserve of } 20 \text { more will be } \\
\text { progressively built after system } \\
\text { start to face mishaps and } \\
\text { possible increases of traffic } \\
\text { (financed with subordinated } \\
\text { stakeholders loans or extra } \\
\text { returns). }\end{array}$ \\
\hline $\begin{array}{l}\text { Payloads (satellite } \\
\text { onboard equipment) }\end{array}$ & $\begin{array}{l}\text { TLC payload in Ka Ku upload download bands capable of meeting } \\
\text { respective planned peaks of user upload download up to ca. 300Gbps } \\
\text { each satellite. Overall per satellite transmitting and receiving capacity } \\
\text { including constellation interlocking, Mission Control and Service } \\
\text { Dataport Ground stations link, stress and emergency factors, goes up to } \\
700 \mathrm{Gbps} .10 \text { satellites are operational at a time, because half of the } \\
\text { constellation is located opposite to the Atlantic Front. } \\
\text { CIR and MIR services separately available at 10Mbps rates upload and } \\
\text { download. Ordinary multiple GNSS devices for orbital routing and } \\
\text { positioning of services. EO medium resolution (2-10m) optical sensors, } \\
\text { mounted on a redundant array of } 3 \text { fixed cameras pointed at different } \\
\text { angles and covering a footage of } 4000 * 3000 \mathrm{Km}, 1 \text { frame / } 10 \text { second } \\
\text { frequency at double level of computerized approximation for resolution } \\
\text { (2 and } 10 \text { meters). Data relay automatic switch with subsequent satellite } \\
\text { optimization. Anti-atmospheric disturbances devices. }\end{array}$ & $\begin{array}{l}\text { Imaging might partly decrease } \\
\text { quality as angle increases when } \\
\text { simultaneous satellite on area } \\
\text { are polarized midway from its } \\
\text { position. Availability of images } \\
\text { is basically for Atlantic - } \\
\text { Mediterranean area and in } \\
\text { daylight or slightly overcast, } \\
\text { although infrared sensors for } \\
\text { night vision are offered. }\end{array}$ \\
\hline $\begin{array}{l}\text { Ground Segment MCC } \\
\text { and SCS (Mission and } \\
\text { Service Control Centers) }\end{array}$ & $\begin{array}{l}4 \mathrm{MCC} \text { and data ports are foreseen, } 2 \text { (with more complex server based - } \\
\text { and big data linked - SCS) are in the West Mediterranean and the South } \\
\text { American Atlantic. The other in tbd locations of the North and South } \\
\text { Pacific so to ensure all over routing visibility access to flying system and } \\
\text { their data connection. }\end{array}$ & $\begin{array}{l}\text { SCS data ports connected to } \\
\text { local mobile networks, Internet, } \\
\text { point to point user front ends. } \\
\text { Big data link is ensured by } \\
\text { dedicated application tapping } \\
\text { from referred and stable open } \\
\text { access data sources [14] to } \\
\text { integrate automatically into } \\
\text { queries and services [15]. }\end{array}$ \\
\hline $\begin{array}{l}\text { Ground Segment user } \\
\text { terminal (on board vessel) }\end{array}$ & $\begin{array}{l}\text { Leased redundant broadcasting and receiving system to microsats in Ka } \\
\text { Ku band based on autotracking signal. For maritime users, } \\
\text { complementing application console for allocation of services and } \\
\text { dedicated applications (AIS, point to point configuration, integrated } \\
\text { media - big data, marine route assistant, telemedicine, EO server), wi-fi } \\
\text { and cabled local connection to cover ship perimeter for the number of } \\
\text { authorized users foreseen in the contract and the relative privileges } \\
\text { (access to CIR service, access to point to point, access to operational } \\
\text { services, etc.). Customized insitu navigation sensors. For land TLC user } \\
\text { standard backhaul tower and management console (Community users) or } \\
\text { set of movable antennas (professional, private, PRS, CBRNe users). }\end{array}$ & $\begin{array}{l}\text { For maritime users optional } \\
\text { access to open source console } \\
\text { middleware to configure specific } \\
\text { services assuming client's own } \\
\text { responsibility. Optional } \\
\text { availability at a price, also, of } \\
\text { underwater internet connected } \\
\text { devices (also submersible sensor } \\
\text { drones). }\end{array}$ \\
\hline
\end{tabular}

The here above EO additional service is connected to an important and inspirational commitment of SHST toward environment, just like for the aforementioned debris risk mitigation devices deployed on the satellites. In fact, all maritime SHST clients subscribe an obligation to make themselves available for EO random visual control against sea pollution, as SHST participates in the SeaBOS (SeaBOS is the Seafood Business for Ocean Stewardship Initiative, a Protocol started in Sweden in 2015 and formally signed one year after by some of the world's leading Ocean seafood corporations, pledging to conscious and sustainable / renewable use of marine resources and their integrity.) Protocol.

Going more into detail with a standard potential Nigerian CBRNe - like management user (e.g. a coastguard or other medium sized vessel), the pattern of services and relative prices could be those in the "workships" cluster, that is a basic TLC web connection at $10 \mathrm{Mbps}$ at Committed Information Rate-CIR conditions (Ka-Ku bands) in upload and download, provided by an included leased and installed signal autotrackingka-ku redundant rec.-transmitter, for a yearly fee of $28 \mathrm{~K} €$. This bundle would comprise a ceiling package of streaming images ( $1 / 10$ seconds) up to 20,000 / year anywhere 
by quadrants of $4000 * 3000 \mathrm{~km}$ at 3 different angles of medium resolution (optionally 2 and 10 meters). Such package is designed to support a 15 people crew, with 3 logins for dedicated P2P, internet big data interaction and Telehealth and emergency services.

Regarding potential Nigerianl and users it must be firstly cleared up, that because of the actual prevailing maritime use of SHST, over the Atlantic Ocean, the geographical slots that can be serviced are limited. These areas will be presumably offered to local investors willing to backhaul the speed band satcomsignal over horizon free perimeters (university campuses, rural communities, etc.), or to local PRS managers including those performing CBRNe- like services, using smaller deployable rec.-transmitters. There are only 2 such slots (out of 10 available over the whole Eastern Atlantic Region up to $2500 \mathrm{~km}$ from the Coast), at para - equatorial latitudes, and therefore interesting for Nigeria. One, coastal, with a limited service capacity due to the proximity to the sea, and the second inland up to 2,000kilometers. In these areas, a full deployment of the satellites signal capacities (300Gbps), could provide minimum $10 \mathrm{Mbps}$ speed band in upload and download mode to a maximum of 20.000 single users of web media connection, for a per area coverage price of ca. $1 \mathrm{M} €$ year to correspond to the SHST Supplier (in turn based on a $50 € /$ year per final user fee component). It is clear, starting from these figures, that a local investor in this area can have a large profit margin, because the selling price of web connections can start from $100 €$ year and even more for professional and community services. The cost of backhaul towers (if more than one) and their operation anyway would beat the charge of the local investor-provider.

It must be noted however that these local providers are likely to join the equity in the purpose company (the SHST Supplier) and therefore, apart from these competitive connection costs, they will gain from rather high financial cash flow revenue rates: $\mathrm{NPV}=95 \%, \mathrm{IRR}=32 \%$ (see the matrix in the last page).

But again, regarding land PRS and CBRNe- like managers, one third of the capacity could be available, which, as for the inner and bigger quadrant in Nigeria, corresponds to ca. 100 connected ( $A$ dividing factor of 10 on the average user absorption has been applied considering the peculiar robustness and characteristics that these services must have because they operate in emergency for life conditions.) units each with 10 logins, at CIR rate, of $10 \mathrm{Mbps}$ upload and download (e.g. service vehicles with 10 staff each), equipped with a leased set of two light, self powered, self tracking $\mathrm{Ka} . \mathrm{Ku}$ band rec.-transmitters, deployable on the scenario (additional antennas will have a cost). The service fee for this is $28 \mathrm{~K} €$ yearly per Unit. To integrate the land service package, unlike the general area, here, given the authorized PRS profile of these users, EO services are supplied, with a similar ceiling package of streaming 2-10 m resolution images a year as in the case of maritime clients. Consider that these images are suitable for emergency management like biochemical threats such as fumes, greenhouse gas masses and the relative aerosol and surface wind spread, but not for radiation detection. Console services, unlike for maritime users, are not included for these land operators, apart from Telemedicine, but they can suitably implement operational on-field Protocols such as SPIDER, etc. Our enquiries revealed and interest here, to fulfil the available slots, both from police public forces and from private networks in the industrial sector, as well as from tourism, for monitoring adventure tours.
To conclude, and coming back to our main starting assumption, it is undoubtedly necessary to get started on any such initiative, by building a minimum level of technological culture in the local provider organization, as it will have to take care by himself of the good functioning of its leased/extended backhaul system, both at the level of hardware, and at the level of console applications and context conditions. The contacts already occurred with Nigerian entrepreneurs during our study indicate a substantial interest, especially in backhauling internet radio rather than media, as it would be more culturally compatible in some rural areas, along with a request for professional and financial web services elsewhere. Interest for maritime operators emerged too. A possible IFI financed programme in Nigeria could tackle both areas; as to the first, for instance the related cultural problems of contents in the internet media could be tackled, and the way to circulate them progressively to prepare the population, making it acceptable to muslim, tribal, rural communities. Because this, and not only in Nigeria, seems to be one of the major obstacles to the propagation of these services. Regarding the second area, the importance of increased sea and land safety and security should also be highlighted as a crucial factor for the Country's growth chances in a context of international cooperation.

\subsection{SHST project financing initiative simulation - accountancy details}

Following the "project financing" principles previously illustrated, Figure 3 here contains a cash flow matrix of the actual SHST model initiative, the one used in our simulation, which in turn incorporates the results of spreadsheets of costs, customer types and numbers, and the relative expected sales. The following are some clearups of economic terms which appear in this Figure, other than the general financial ones already described: a) "current expenditure" is meant as inclusive of long term costs such as launch and relative insurance premium, development of server and application software, all levels of maintenance and side services required by the system (i.e. the remote medical assistance); b) "capital expenditure" is only covering the "build" phase construction for the ground (physical) and flying system, whereas "ground system" is referring to the terrestrial infrastructure, and includes the leased dedicated vessel terminal equipment complete with integrated software and applications, as well as the backhaul system (not exceeding one unit) or other user terminals for the different clusters of land users.

The Figure's foot page box bears a list of some crucial factors in the calculations and the final results of the indexes NPV and IRR (NPV (Net Present Value) is the value of the expended capital investment compensated by the net returns of the project along its duration. All of the cash flow yearly balances are to this purpose to be discounted at an ideal comparative reference rate for the sector (the reasonable rate of return for an alternative investment). It is often presented as a percentage of the capital investment. The larger this percentage the higher is its banking attractiveness.

IRR (Internal Rate of Return) is the rate (not predefined as above) at which the two values above inserted in a cash flow settlement scheme (the capital investment and its net returns) can be discounted to become 0 . The value obtained is a rate of return for the investment which can be compared to what is obtainable for capital remuneration on the market and defines therefore its attractiveness for investors. 
Both rates can be expressed in "economical" and "financial" terms, the latter differentiated by the inclusion of all financing items, that is the financial capital and its relative interests. This second index is usually lower as it takes already into account the fixed rate of return incorporated by the banks in their project loan conditions.): the debt / equity ratio and their absolute values, the financial interest rate, the forfait taxation rate. The latter is applied as if the initiative should be set up in Malta and it is comprehensive of both that direct and indirect taxation.

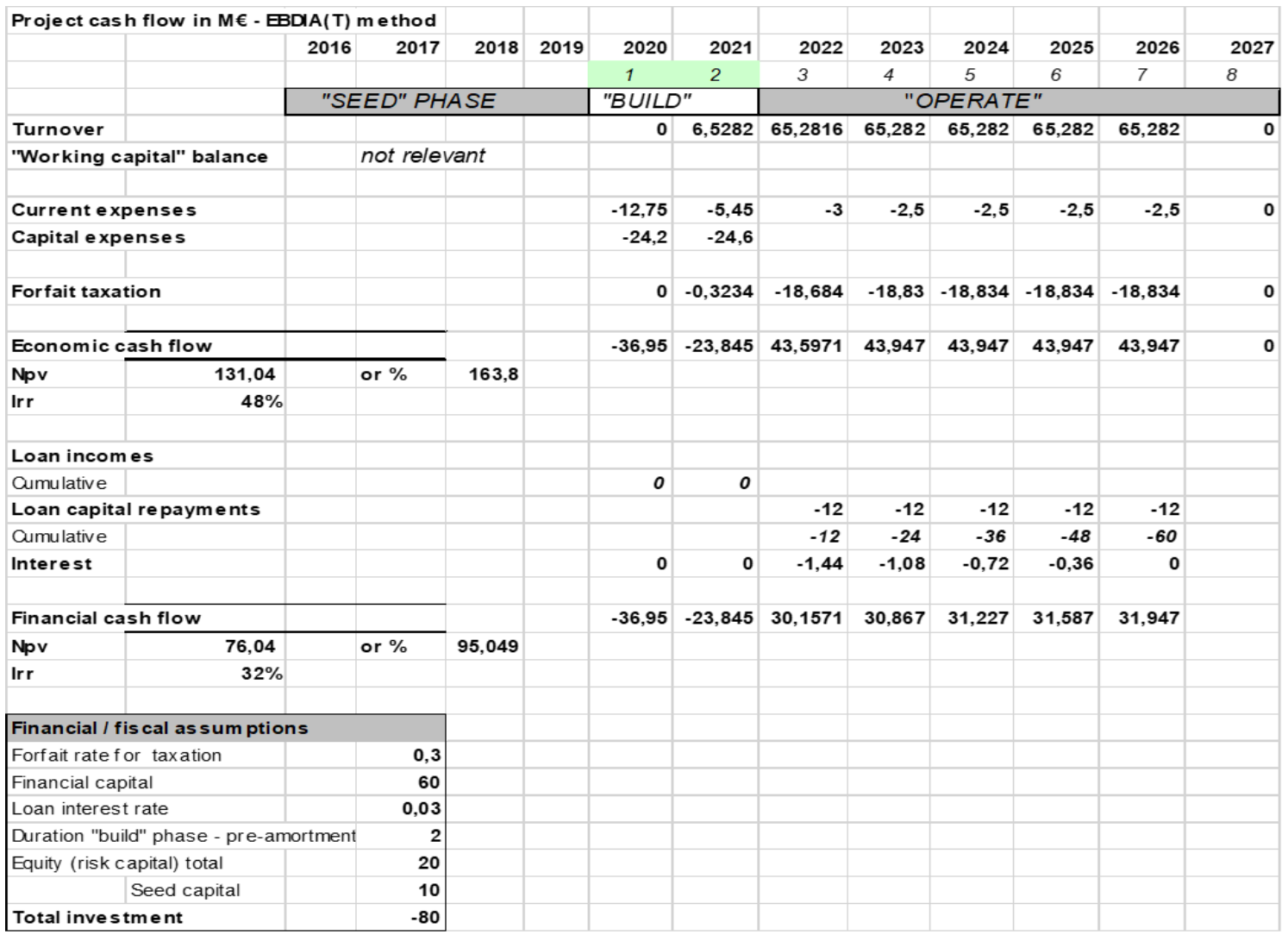

Figure 3. Elements of a potential project financing scheme for the SHST microsat system proposal

\section{CONCLUSIONS}

We believe that such a comprehensive vision of the process to implement these innovative satellite services in the Developing Countries, from local context preparation to the step by step Service configuration with a desing to cost / price definition, can really offer to all local potential users, and especially those in the Emergency Management field, an useful operational and comparative benchmark.

Also, the Model approach of the SHST - so far only theoretical - satellite system, can best involve them into further discussions with our research group on their specific needs and situations, aiming at a testing and designing sustainable variations in the Model.

From the information we are starting to receive regarding the service prices of new small satellite TLC services coming to the market, we are also convinced that the characteristics of SHST, based on a small constellation with sustainability concerns and implementations, along with its private - project financing approach, makes it competitive in its class, and therefore has increased its chances to aggregate the critical mass of committed users, which will make its launch possible.

\section{REFERENCES}

[1] Sciortino, G., Fotia, R., Sumah, K. (2019). Satellite TLC for the reduction of digital divide and socio economic inclusion. Pegaso International Conference 2019, Social Inclusion, Education, and Digital Society, Malta.

[2] Denis, G., Claverie, A., Pasco, X., Darnis, J.P., Maupeou, B., Lafaye, M., Morel, E. (2017). Towards disruptions in Earth observation? New Earth Observation systems and markets evolution: Possible scenarios and impacts. Acta Astronautica, 137:

415-433. https://doi.org/10.1016/j.actaastro.2017.04.034

[3] Colton, K., Klofas, B. (2016). Supporting the flock: Building a ground station network for autonomy and reliability. $30^{\text {th }}$ Annual AIAA/USU Conference on Small Satellites.

[4] Berrick, S. (2020). Commercial Smallsat data acqusition program CSDAP. Washington, USA: www.nasa.gov.

[5] Euroconsult. (2019). Small Satellite Market 2019. Euroconsult, Paris, France.

[6] Christopherson, J. (2019). Land Remote Sensing Satellite Compendium. Department of Interior - Joint Agency for Commercial Imagery Evaluation, Washington USA.

[7] Bergamini, E., Jacobone, F., Morea, D., Sciortino, G.P. (2018). The increasing risk of space debris impact on earth: Case studies, potential damages, international liability framework and management systems. Enhancing CBRNE Safety \& Security: Proceedings of the SICC 2017 Conference, pp. 271-280. https://doi.org/10.1007/978-3-319-91791-7_31

[8] Sciortino, G. (2017). New insurance models evolve with 
the development of commercial space economy. International Astronautical Federation - Adelaide IAC 2017 E.3.6, Paris.

[9] OECD. (2018). Development Cooperation Report Joining forces to leave non one behind. OECD, Paris.

[10] Sumah, K. (2018). Applying the Diamond approach to the competitiveness of African space industry. Thesis TUM School of Management - Technische Universitaet Munchen, Munchen.

[11] Sciortino, G., Gregorio, A., Puglia, S., Ferroni, P. (2018). Space services for sea fleets emerge as an elective private market for the Italian small satellites platforms. International Astronautical Federation IAC Bremen 2018 E3.3 Symposium, Paris.

[12] Imperatori, G. (1995). Il project financing, una tecnica, una cultura.Il Sole 24 Ore, Milan.

[13] Marsi, S., Gregorio, A., Maris, M., Puligheddu, M. (2017). PicoAgri. Realization of a low-cost, remote sensing environment for monitoring agricultural fields through small satellites and drones. 40th International Convention on Information and Communication Technology, Electronics and Microelectronics (MIPRO), Opatija, 2017, pp. 1073-1078. https//doi.org/10.23919/MIPRO.2017.7973583

[14] Sciortino, G.P., Indennidate, D., Sorace, R., Puglia, S., Bergamini, E. (2017). Is the market of satellite earth imagery capable of supporting new investments and launches under project financing models? Proceedings of 68th International Astronautical Congress: Unlocking Imagination, Fostering Innovation and Strengthening Security, IAC 2017, Adelaide, Australia, 25 September 2017, pp. 11966-11977.

[15] Mirovic, M., Milicevic, M., Obradovic, I. (2018). Big Data in Maritime Industry. Nase More: International Journal of Maritime Science \& Technology, 65(1): 5672. https://doi.org/10.17818/NM/2018/1.8

[16] Sozzi, M., Marinello, F., Pezzuolo, A., Sartori, L. (2018). Benchmark of satellites image services for precision agricultural use. In Proceedings of the AgEng Conference, Wageningen, The Netherlands, pp. 8-11.

\section{NOMENCLATURE}

\section{Acronyms}

COTS Commercial Off the Shelf

DAC Development Assistance Committee OECD

IFI International Financial Institutions

IDI ICT Development Index

ITU International UN Telecommunications Union

LDC Least Developed Countries

ODA Official Development Assistance 